\title{
Genome-wide profiling of long non-coding RNA expression patterns in anthracycline-resistant breast cancer cells
}

\author{
DONG-XU HE ${ }^{1 *}$, GUANG-YUAN ZHANG ${ }^{1 *}$, XIAO-TING GU ${ }^{2 *}$, AI-QIN MAO ${ }^{2}$, \\ CHUN-XIAO LU ${ }^{2}$, JIAN JIN ${ }^{2}$, DE-QUAN LIU ${ }^{3}$ and XIN MA ${ }^{2}$ \\ ${ }^{1}$ National Engineering Laboratory for Cereal Fermentation Technology; ${ }^{2}$ Department of Cellular and Molecular Pharmacology, \\ School of Medicine and Pharmaceutics, Jiangnan University, Wuxi, Jiangsu 214122; ${ }^{3}$ Department of Breast Surgery, \\ The Third Affiliated Hospital, Kunming Medical University, Kunming, Yunnan 650031, P.R. China
}

Received June 12, 2016; Accepted July 29, 2016

DOI: $10.3892 /$ ijo.2016.3665

\begin{abstract}
Long non-coding RNAs (lncRNAs) are involved in cancer progression. In the present study, we analyzed the lncRNA profiles in adriamycin-resistant and -sensitive breast cancer cells and found a group of dysregulated lncRNAs in the adriamycin-resistant cells. Expression of the dysregulated lncRNAs was correlated with dysregulated mRNAs, and these were enriched in GO and KEGG pathways associated with cancer progression and chemoresistance development. Among these 1ncRNA-mRNA interactions, some lncRNAs may cis-regulate neighboring protein-coding genes and be involved in chemoresistance. We then validated that the lncRNA NONHSAT028712 regulated nearby CDK2 and interfered with the cell cycle and chemoresistance. Furthermore, we identified another group of lncRNAs that trans-regulated genes by interacting with different transcription factors. For example, NONHSAT057282 and NONHSAG023333 modulated chemoresistance and most likely interacted with the transcription factors ELF1 and E2F1, respectively. In conclusion, in the present study, we report for the first time the IncRNA expression patterns in adriamycin-resistant breast cancer cells, and provide a group of novel lncRNA targets that mediate chemoresistance development in both cis- and trans-action modes.
\end{abstract}

Correspondence to: Dr Xin Ma, Department of Cellular and Molecular Pharmacology, School of Medicine and Pharmaceutics, Jiangnan University, Li Hu Street, Wuxi, Jiangsu 214122, P.R. China E-mail:maxin@jiangnan.edu.cn

Dr De-Quan Liu, Department of Breast Surgery, The Third Affiliated Hospital, Kunming Medical University, People's West Road, Kunming, Yunnan 650031, P.R. China

E-mail: dequanliu08@163.com

*Contributed equally

Key words: long non-coding RNAs, cancer chemoresistance, breast cancer, cis-regulation, trans-regulation

\section{Introduction}

Long non-coding RNAs (lncRNAs) are encoded similarly to coding genes but do not contain a protein-coding sequence. The transcripts of lncRNA are $>200$ nucleotides long and expressed at lower levels than protein-coding transcripts. Some lncRNAs directly modulate gene expression in a cis manner; these lncRNAs may be expressed from the promoter (1), intron (2) and enhancer regions (3) of certain genes and then regulate neighboring protein-coding genes on the same chromosome. Furthermore, some lncRNAs distally regulate gene expression across multiple chromosomes in a trans manner. These lncRNAs can facilitate enhancer function via long-range DNA looping interactions (4). Also, some lncRNAs appear to modulate the DNA-binding of certain transcription factors (TFs) and non-DNA-binding cofactors at several target sites, thus affecting gene expression in trans (5).

In recent years, several lncRNAs have been implicated in cancer development and progression (6-8). Chemoresistance is an important feature of cancer progression, since chemoresistant cells are insensitive to the apoptotic signals delivered by cytotoxic chemotherapeutic agents. They also show a strong ability to proliferate. A large degree of chemoresistance is acquired during the response of cancer cells to unfavorable niches, and their rapid response depends on the effective and heritable epigenetic regulation of gene expression, including DNA methylation and microRNA regulation (9). For example, methylation of microRNA-200c inhibits microRNA expression, so the targets of the microRNA, including modulators of epithelial mesenchymal transition pathways that essentially promote cell proliferation and diminish apoptosis, are activated (10-14). In the current decade, emerging evidence indicates that $70-90 \%$ of the mammalian genome produces lncRNAs, which are another important component of epigenetic regulation $(15,16)$. However, although $>10,000$ mammalian lncRNAs have been catalogued (5), functional studies are as yet limited in number, and how the network of lncRNAs is involved in cancer chemoresistance still requires elucidation.

In the present study, we set out to analyze lncRNA expression in adriamycin-resistant breast cancer cells using microarrays, and compare their lncRNA expression profile 

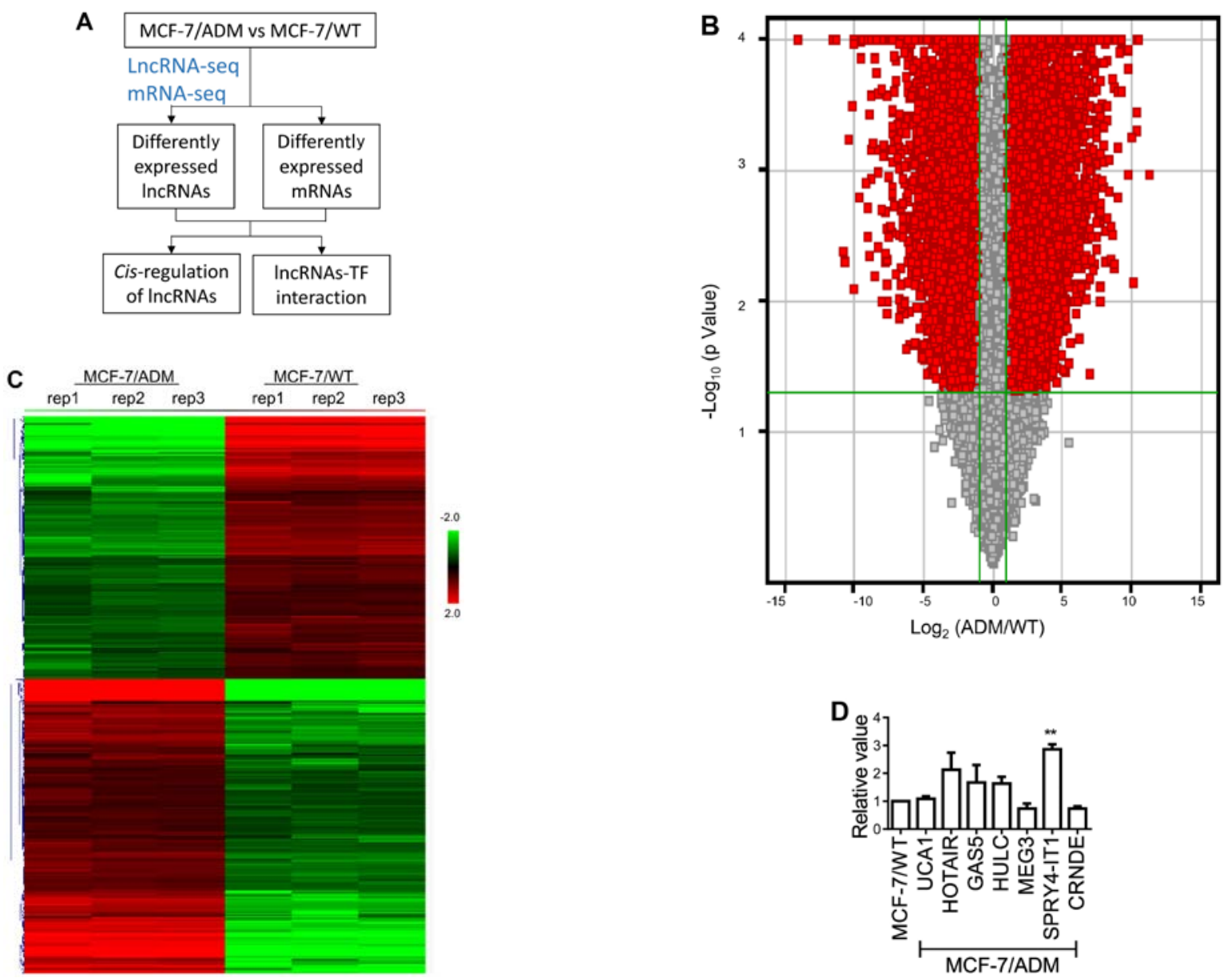

Figure 1. LncRNA and mRNA profiling in MCF-7 cells. (A) Work flow of this study. (B) Volcano plot of differentially-expressed lncRNAs. Profiling was replicated 3 times. (C) Hierarchical clustering of distinct mRNAs in MCF-7/ADM and MCF-7/WT cells. Profiling was replicated 3 times. (D) RT-PCR validated the expression of cancer-related lncRNAs. The values of all lncRNAs in MCF-7/WT cells were normalized to 1.

with that of parental chemosensitive cells in order to identify and characterize dysregulated lncRNAs that may be involved in breast cancer chemoresistance (Fig. 1A).

\section{Materials and methods}

Cell culture. MCF-7/WT human breast cancer cells were obtained from the American Type Culture Collection (ATCC; Manassas, VA, USA). Adriamycin (ADM)-resistant cells (MCF-7/ADM) and paclitaxel (PTX)-resistant cells (MCF-7/ PTX) were derived by treating MCF-7 cells with stepwise increasing concentrations of ADM or PTX over 8 months (17). The cells were cultured in RPMI-1640 supplemented with $10 \% \mathrm{FBS}, 100 \mu \mathrm{g} / \mathrm{ml}$ penicillin and $100 \mathrm{U} / \mathrm{ml}$ streptomycin.

Microarray. The microarray profiling was conducted in the laboratory of OE Biotechnology Co. (Shanghai, China). RNA from MCF-7/ADM and MCF-7/WT cells was separately extracted using the acid-phenol and chloroform method. Cyanine-3-CTP-labeled cRNA was obtained using a Quick Amp Labeling kit (Agilent Technologies, Santa Clara, CA, USA) and then purified with an RNeasy Mini kit (Qiagen, Valencia, CA, USA). The labeled cRNAs were then hybridized onto Agilent-062918 OE Human lncRNA Microarray V4.0 028004 (Agilent Technologies), which is a Custom Gene
Expression Array for OE Biotechnology Co. and detects 46,506 lncRNAs. After washing, the arrays were scanned with an Agilent scanner (G2505C).

Quality control of the microarray data. Total RNA was quantified by the NanoDrop 2000 (Thermo Fisher Scientific, Waltham, MA, USA) and the RNA integrity was assessed using Agilent Bioanalyzer 2100 (Agilent Technologies). RNA with RNA integrity number (RIN) value $>7$ and $28 \mathrm{~S} / 18 \mathrm{~S}>0.7$ was used for microarray analysis. After the raw data extraction, the median CV (\%) for each probe set was reported as the array reproducibility. To analyze the biological repeatability of the microarrays between MCF-7/ADM ( $n=3)$ or MCF-7/WT $(n=3)$ cells, two-dimensional principal component analysis was performed.

Data deposition. The microarray data have been submitted to GEO (GSE81971).

Data analysis. Raw data of microarray was generated using Agilent Feature Extraction software (Agilent Technologies) and then normalized using GeneSpring's quantile normalization (version 12.5; Agilent Technologies). Differentially-expressed lncRNAs were identified with a fold change $\geq 2.0$ and a P-value $<0.05$. WebGestalt (http://bioinfo.vanderbilt.edu/webgestalt/) 
was used for Gene Ontology (GO) and Kyoto Encyclopedia of Genes and Genomes (KEGG) pathway enrichment analysis.

Real-time PCR. LncRNA expression was analyzed using qRT-PCR. Briefly, total RNA was extracted from MCF-7/WT and /ADM cells with TRIzol (Invitrogen, Carlsbad, CA, USA). cDNA was synthesized from total RNA ( $3 \mu \mathrm{g})$ using the SuperScript First Strand Synthesis system (Invitrogen) with Oligo (dT) primers. Primers used for real-time PCR were as previously described for UCA1 $(18,19)$, HOTAIR (6), GAS5 (20), HULC (21), MEG3 (22), SPRY4-IT1 (23) and CRNDE (24). Primer for NONHSAT028712 was: forward, 5'-AAATACCTCACCCTCATCTATACCAAC-3' and reverse, 5'-TTTCCCGTTGCCATTGAT-3'; for NONHSAT057282 was forward, 5'-AGCCGGAGGTGAGGAAGTT-3' and reverse, 5'-A AGATTTTATTAGATTTTGGAACCTGAG-3'; for NONHSAG023333 was forward, 5'-GTTGGGAAATCAAG CATCGT-3' and reverse, 5'-TTTAGCAAAAATGCAACTA CATCC-3'.

The RT-PCR values were normalized to GAPDH and calculated using the $2^{-\Delta \Delta \mathrm{CT}}$ method.

LncRNA inhibition and functional studies. LncRNA Smart Silencer was synthesized by Guangzhou RiboBio Co., Ltd., (Guangzhou, China) and used to inhibit lncRNA. The inhibitor was then transfected into MCF-7 cells using Lipofectamine (Invitrogen).

MCF-7/ADM or MCF-7/PTX cells transfected with lncRNA inhibitor were seeded onto 96-well plates and exposed to different concentrations of ADM or PTX for $48 \mathrm{~h}$. Then cell viability and $\mathrm{IC}_{50}$ were assessed as previously described $(25,26)$.

In experiments analyzing the cell cycle, MCF-7/ADM cells transfected with lncRNA inhibitor were fixed and stained with $100 \mu \mathrm{g} / \mathrm{ml}$ propidium iodide (PI; Sigma Life Science) containing RNase. PI fluorescence was detected using a FACSCalibur flow cytometer on the PE-Texas Red channel for DNA content.

\section{Results}

Expression of lncRNAs in chemoresistant breast cancer cells. To gain insights into the role of lncRNAs in chemoresistance, we used microarray-based profiling to analyze the lncRNAs and mRNAs in adriamycin-resistant MCF-7/ADM cells and their chemosensitive parental control MCF-7/WT cells (17). In MCF-7/ADM cells, 4030 lncRNAs and unannoted transcripts were upregulated and 3708 were downregulated (Fig. 1B; Submitted online as GSE81971), while 3423 mRNAs were upregulated and 2950 were downregulated (Fig. 1C; fold-change $\geq 2, \mathrm{P}<0.05$ ), suggesting that lncRNAs may be dysregulated and participate in the development of chemoresistance.

We then validated several cancer-related lncRNAs with RT-PCR (6,7,18-24). Among these, SPRY4-IT1 was upregulated in chemoresistant MCF-7/ADM cells by both microarray analysis and RT-PCR, suggesting that it may play a role in chemoresistance; other IncRNAs were not significantly changed either in microarray analysis or RT-PCR (Fig. 1D). These data, thus, confirm the accuracy of microarrays, while
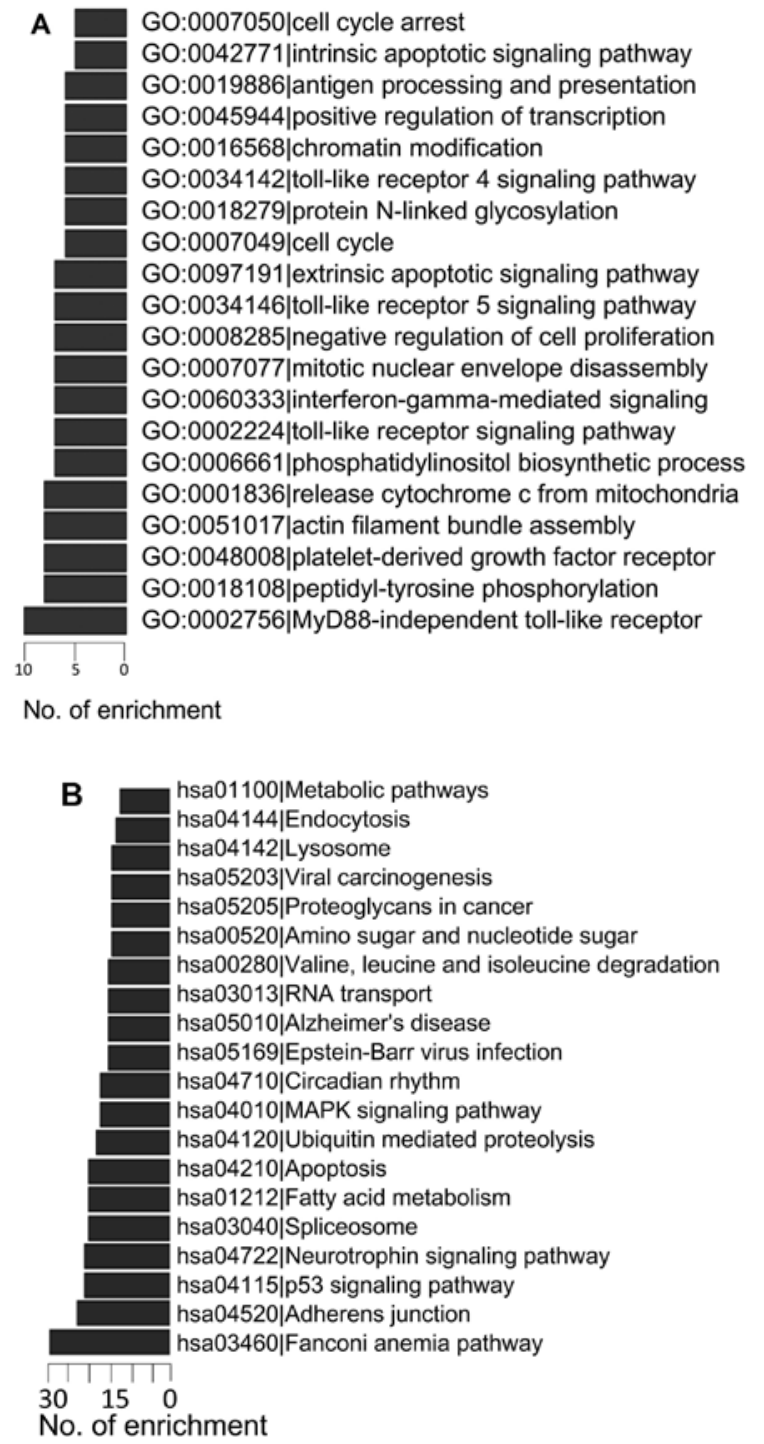

Figure 2. Function analysis. The mRNAs that were correlated with the 200 most markedly changed lncRNAs were analyzed in GO and KEGG. Bar graphs show (A) the top 20 enriched GO processes and (B) KEGG pathways.

prompted us to search for new lncRNAs that may mediate chemoresistance.

In order to identify new candidate IncRNAs in chemoresistance, we then chose the top 200 most significantly changed lncRNAs for further analysis.

LncRNAs correlate with mRNA expression. LncRNAs influence gene expression by regulating chromatin remodeling, transcription and post-transcriptional processing (5). To identify potential lncRNA targets, we calculated the Pearson correlation of each significantly changed lncRNA with each significantly changed mRNA. An lncRNA and an mRNA were considered to be correlated when the coefficient was $>0.7(\mathrm{P}<0.05)$, so such mRNAs might be regulated by their correlated lncRNAs. The most correlated mRNAs for top 10 changed lncRNAs are exemplified in Table I.

The 500 lncRNA-mRNAs with the highest Pearson correlation coefficient values were chosen for functional analysis in GO and KEGG using the method described by Guttman et al (27). The GO and KEGG functions of each 
Table I. Top 10 changed lncRNAs and their correlated mRNAs.

\begin{tabular}{|c|c|c|c|c|}
\hline LncRNA $^{a}$ & $\mathrm{FC}(\mathrm{abs})^{\mathrm{a}}$ & $\mathrm{Up} /$ down $^{\mathrm{a}}$ & Location & Top 3 correlated mRNA (P-value; coefficience) \\
\hline NONHSAT082326 & 16783 & Down & $\begin{array}{c}\operatorname{chr} 21: 43782390 \\
-6644\end{array}$ & $\begin{array}{l}\text { SLC30A1 }(0.00010002310686108 ; 0.991822939253643) \\
\text { FKBP1A }(0.00010007371243696 ;-0.991820868137818) \\
\text { PRDX2 (0.000100126235187625;0.991818719110682) }\end{array}$ \\
\hline ENST00000455354 & 3060 & Down & $\begin{array}{c}\operatorname{chr} 21: 41755010 \\
-7285\end{array}$ & $\begin{array}{l}\text { LAMB2 }(0.000100025125846499 ;-0.991822856613367) \\
\text { DENND2C }(0.000100084970897196 ; 0.991820407437975) \\
\text { USP2 (0.000100086690841299;-0.991820337059573) }\end{array}$ \\
\hline ENST00000422749 & 2703 & Down & $\begin{array}{c}\operatorname{chr} 21: 41755010 \\
-7285\end{array}$ & $\begin{array}{l}\text { NEBL }(0.000100168472873419 ; 0.991816991316235) \\
\text { ABLIM1 }(0.000100266459849063 ; 0.991812984413778) \\
\text { C17orf51 (0.000100447057119302;-0.99180560450735) }\end{array}$ \\
\hline ENST00000444046 & 2522 & Down & $\begin{array}{c}\operatorname{chr} 21: 41755010 \\
-7285\end{array}$ & $\begin{array}{l}\text { ANPEP }(0.000100005971417187 ;-0.991823640668079) \\
\text { KIF23 }(0.000100364967970128 ;-0.991808958168178) \\
\text { ARL6IP5 }(0.000100426675196719 ; 0.991806437060535)\end{array}$ \\
\hline NONHSAT128425 & 1756 & Down & $\begin{array}{c}\text { chr8: } 120221107 \\
-55888\end{array}$ & $\begin{array}{l}\text { GDA }(0.00010028234061338 ;-0.991812335198366) \\
\text { GHITM }(0.000100331837803225 ; 0.991810312051648) \\
\text { MRPL13 }(0.000100359825255418 ;-0.991809168313582)\end{array}$ \\
\hline NONHSAT023895 & 2501 & Up & $\begin{array}{c}\text { chr11: } 102667774 \\
-8070\end{array}$ & $\begin{array}{l}\text { UBXN8 }(0.000100363702178807 ; 0.991809009891383) \\
\text { SSH1 }(0.000100433168514824 ; 0.991806171814755) \\
\text { NUP188 }(0.000100566546204432 ;-0.991800725355188)\end{array}$ \\
\hline NONHSAT022443 & 894 & Up & $\begin{array}{c}\text { chr11:67353574 } \\
-910\end{array}$ & $\begin{array}{l}\text { HSCB }(0.000100041635865034 ;-0.991822180863287) \\
\text { INPPL1 }(0.000100075383041166 ;-0.991820799774519) \\
\text { SNTB2 }(0.000100118418071562 ;-0.991819038921128)\end{array}$ \\
\hline NONHSAT091446 & 621 & Up & $\begin{array}{c}\text { chr3: } 120123741 \\
-30173\end{array}$ & $\begin{array}{l}\text { PTP4A1 (0.000100232420515941;-0.991814376135381) } \\
\text { DPY19L3 (0.000100276937161676;-0.991812556089039) } \\
\text { LOC100129846 (0.000100350020328222;-0.9918095689) }\end{array}$ \\
\hline NONHSAT023896 & 574 & Up & $\begin{array}{c}\text { chr11:102668127 } \\
-877\end{array}$ & $\begin{array}{l}\text { ACSS3 (0.000100063079112198;-0.991821303280653) } \\
\text { RYBP (0.000100092305234907;-0.991820107328388) } \\
\text { ZNF57 (0.000100124371169228;-0.991818795369462) }\end{array}$ \\
\hline NONHSAT005455 & 531 & Up & $\begin{array}{c}\text { chr } 1: 117282602 \\
-5231\end{array}$ & $\begin{array}{l}\text { BACE1 (0.000100119326125993;-0.991819001770562) } \\
\text { RBMS1 }(0.000100213122112613 ; 0.991815165268592) \\
\text { NEXN }(0.000100296526373889 ; 0.991811755319101)\end{array}$ \\
\hline
\end{tabular}

${ }^{a}$ More information for the lncRNA can be found in online dataset (GSE81971); FC(abs), absolute difference for fold change value; Up/down, MCF-7/ADM vs. MCF-7/WT.

IncRNA-correlated mRNA were analyzed, then a hypergeometric cumulative distribution function was applied to calculate the enrichment of functional terms in the annotation of these mRNAs. The most enriched GO processes and KEGG pathways are shown in Fig. 2. Both analyses showed that pathways directly associated with apoptosis and cell proliferation were frequently regulated by lncRNAs, including release of cytochrome $c$ from mitochondria (GO:0001836), cell cycle (GO:0007049), cell cycle arrest (GO:0007050), apoptosis (KEGG 04210), negative regulation of cell proliferation (GO:0008285), MAPK signaling pathway (KEGG 04010) and p53 signaling pathway (KEGG 04115).

Cis-regulation of InRNAs. Cis-regulation by IncRNAs of their correlated mRNAs was then analyzed in chemoresistant
MCF-7/ADM cells vs. MCF-7/WT cells. Several lncRNAs were located $100 \mathrm{~K}$ windows upstream or downstream of the given mRNA, and the mRNA expression was significantly correlated with the lncRNA. Possible cis-regulation of their correlated mRNAs is exemplified in Table II. Among these, we found that NONHSAT028712 was significantly increased in MCF-7/ADM cells (Table II and Fig. 3A). We also analyzed the expression of NONHSAT028712 in another chemoresistant breast cancer cell line MCF-7/PTX, which is paclitaxel-resistant (9). This lncRNA also significantly increased in MCF-7/ PTX cells (Fig. 3A). These data suggest a possible role of NONHSAT028712 in mediating chemoresistance.

The cis-regulation of NONHSAT028712 is shown in Fig. 3B. Genes of several significantly-changed mRNAs was found to locate near the coding sequence of NONHSAT028712. 

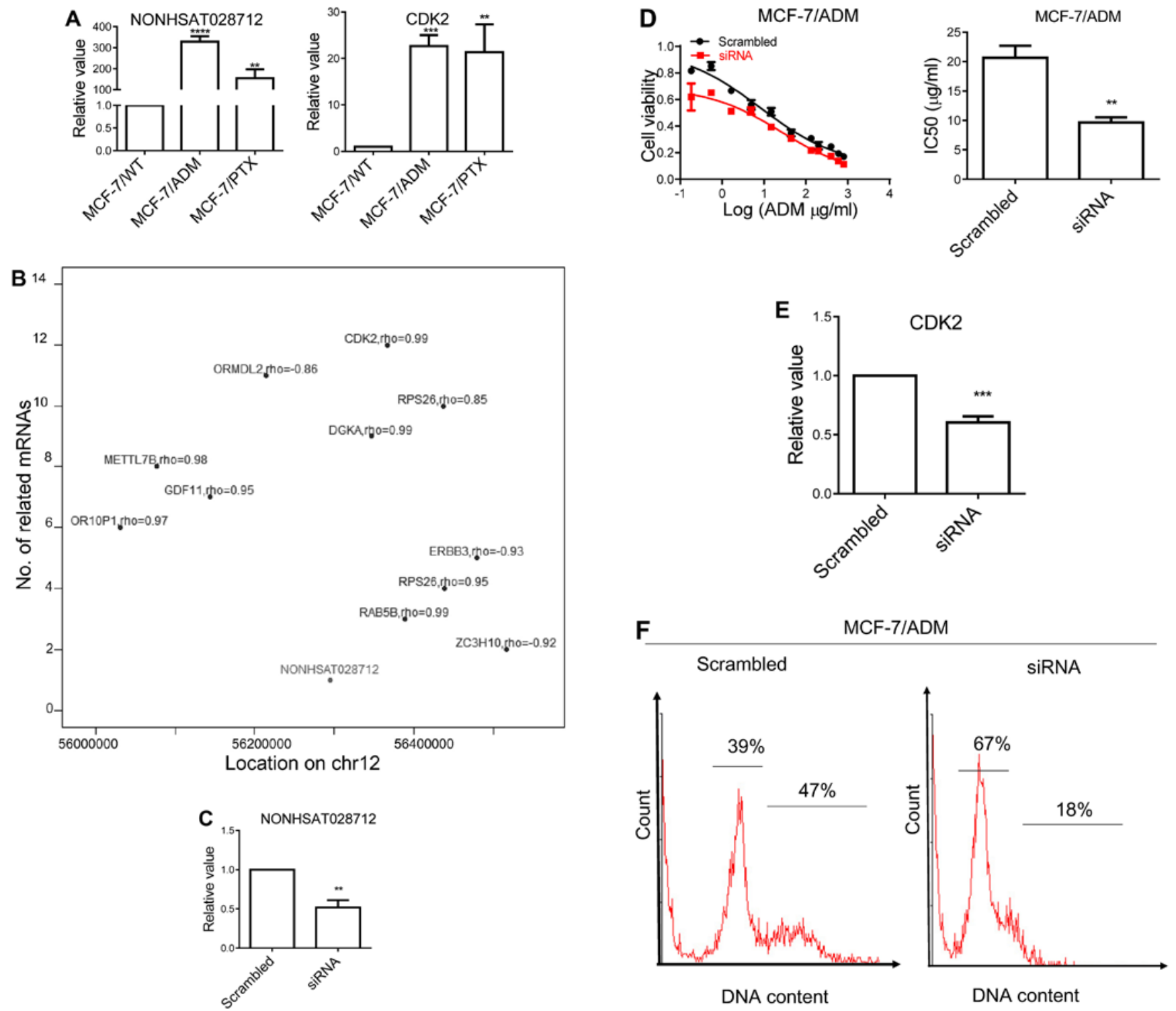

Figure 3. Cis-regulation by lncRNAs. (A) Expression of the lncRNA NONHSAT028712 and CDK2 was higher in MCF-7/ADM and MCF-7/PTX cells as analyzed with RT-PCR. Values were calculated by the $2^{-\triangle \Delta C T}$ method; the values for MCF-7/WT cells were normalized to 1 . (B) Cis-regulatory map of the IncRNA NONHSAT028712 on chromosome 12. (C) Expression of NONHSAT028712 was analyzed via RT-PCR after knockdown by siRNA in MCF-7/ADM cells. Values were calculated by the $2^{-\Delta \Delta C T}$ method. (D) Chemoresistance of MCF-7/ADM cells was inhibited by NONHSAT028712 siRNA. Cell viability was analyzed using MTT assays, and the $\mathrm{IC}_{50}$ was determined by non-linear regression. (E) CDK2 mRNA level was decreased when MCF-7/ADM cells were treated with NONHSAT028712 siRNA. (F) Cell cycle arrest in MCF-7/ADM cells were treated with NONHSAT028712 siRNA.

Table II. Cis-regulation of top 10 changed lncRNAs on their correlated mRNAs.

\begin{tabular}{|c|c|c|c|}
\hline $\begin{array}{l}\text { LncRNAs } \\
\text { (downregulated) }\end{array}$ & $\begin{array}{c}\text { Possible targets of } \\
\text { cis-regulation (coefficient) }\end{array}$ & $\begin{array}{l}\text { LncRNAs } \\
\text { (upregulated) }\end{array}$ & Possible targets of CIS-regulation (coefficient) \\
\hline NONHSAT128425 & NOV $(-0.983)$ & NONHSAT022443 & $\begin{array}{l}\text { SSH3 (-0.985); RPS6KB2 (-0.975); RAD9A } \\
(-0.971)\end{array}$ \\
\hline NONHSAT006799 & $\begin{array}{l}\text { PMF1 (0.958); LMNA (-0.9878); } \\
\text { SEMA4A (0.962) }\end{array}$ & NONHSAT028712 & $\begin{array}{l}\text { ZC3H10(-0.917); RAB5B(0.985002614214423); } \\
\text { RPS26 (0.954); OR10P1 (0.972); METTL7B } \\
(0.975) ; \text { DGKA }(0.991) ; \text { CDK2 }(0.986)\end{array}$ \\
\hline NONHSAT042185 & $\begin{array}{l}\text { CTDSPL2 (-0.821); } \\
\text { CASC4 }(0.970)\end{array}$ & NONHSAT098174 & FGF2 (0.943) \\
\hline NONHSAT143304 & CDH3 (0.991) & NONHSAT057176 & RAB12 (0.961) \\
\hline NONHSAT012940 & $\begin{array}{l}\text { CSGALNACT2 (-0.983); } \\
\text { RET }(0.966) ; \text { BMS1 }(-0.977)\end{array}$ & NONHSAT022441 & $\begin{array}{l}\text { RPS6KB2 (-0.968); RAD9A (-0.972); SSH3 } \\
(-0.983) ; \text { ADRBK1 (-0.989) }\end{array}$ \\
\hline
\end{tabular}



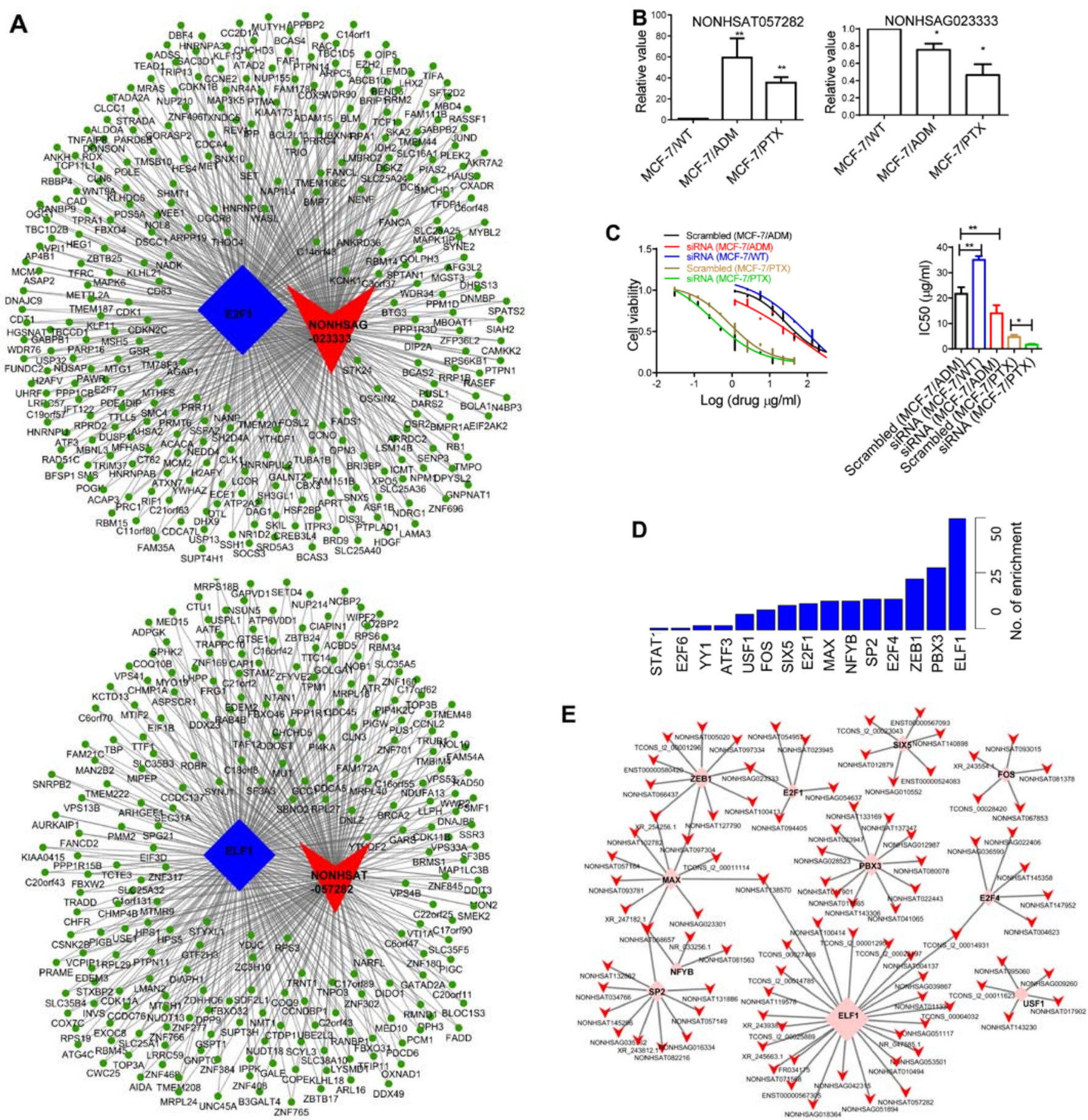

Figure 4. LncRNAs interact with TFs. (A) NONHSAT057282 and NONHSAG023333 interacted with ELF1 and E2F1, and were correlated with 241 and 308 genes in chemoresistant MCF-7/ADM cells vs. MCF-7/WT cells. (B) NONHSAT05728 was upregulated in MCF-7/ADM and MCF-7/PTX cells, while NONHSAG023333 was upregulated in the chemosensitive MCF-7/WT cells as analyzed by RT-PCR. (C) Knockdown of NONHSAT057282 in MCF-7/ADM and MCF-7/PTX cells reversed the chemoresistance to ADM and PTX, respectively, while knockdown of NONHSAG023333 in MCF-7/WT cells increased it. The cell viability was analyzed by MTT assays (left panel), and the $\mathrm{IC}_{50}$ was determined by non-linear regression (right panel). (D) Top $15 \mathrm{TFs}$ with highest enrichment of lncRNAs. (E) Two-element relation graph of the top 100 lncRNA-TF interactions.

Among these mRNAs, the cell cycle kinase CDK2 showed a high mRNA level in MCF-7/ADM and MCF-7/PTX cells (Fig. 3A), and this has been associated with cancer progression and chemoresistance $(28,29)$. We therefore inhibited the expression of NONHSAT028712 with a synthesized inhibitor (Fig. 3C), then analyzed the chemoresistance of the treated MCF-7/ADM cells. The $\mathrm{IC}_{50}$ significantly decreased in MCF-7/ ADM cells when NONHSAT028712 was inhibited (Fig. 3D), along with a lower CDK2 mRNA level (Fig. 3E) and a higher rate of cell cycle arrest in G1 (Fig. 3F). These data strongly suggest that NONHSAT028712 regulates chemoresistance via a CDK2-related pathway, while the mechanism requires further exploration.

Interaction of transcription factors with lncRNAs. To identify the possible role of IncRNA-TF interactions in regulating 
gene expression, we first predicted the TFs of lncRNAcorrelated mRNAs using data from Gerstein et al (30) that showed the genomic binding information of different TFs. Then the intersections of IncRNA-mRNA and mRNA-TF were calculated with a hypergeometric cumulative distribution. Each lncRNA was significantly associated with several TFs (data not shown). For instance, NONHSAT057282 and NONHSAG023333 were significantly correlated with ELF1 and E2F1, and enriched the most mRNAs of all lncRNATF interactions. NONHSAT057282-ELF1 co-regulated 241 genes and NONHSAG023333-E2F1 co-regulated 308 genes. We then drew a ternary relation graph of the two interactions with Cytoscape 3.01 software (Agilent) (Fig. 4A). Furthermore, NONHSAT05728 was upregulated in chemoresistant MCF-7/ADM and MCF-7/PTX cells vs. chemosensitive MCF-7/WT cells, while NONHSAG023333 was upregulated in MCF-7/WT vs. MCF-7/ADM and MCF-7/ PTX cells (Fig. 4B), suggesting that NONHSAT05728 may enhance chemoresistance, but NONHSAG023333 may negatively regulate chemoresistance. Indeed, when we knocked down NONHSAT05728, chemoresistance decreased in both MCF-7/ADM and MCF-7/PTX cells. On the other hand, knockdown of NONHSAG023333 increased the chemoresistance in MCF-7/WT cells (Fig. 4C). Therefore, these results suggest that both NONHSAT05728 and NONHSAG023333 are involved in chemoresistance, and their activity may be facilitated by TFs.

The top 15 TFs with the highest enrichment of lncRNAs are summarized in Fig. 4D, indicating the potential involvement of certain TFs in regulating chemoresistance via lncRNAs. In order to visualize these most significantly-related lncRNA-TF interactions, the top 100 with the lowest Q-values were then used to draw a two-element relation graph (Fig. 4E). ELF1 was still most frequently associated with several lncRNAs, and PBX3 and ZEB1 were also intensively associated with lncRNAs.

\section{Discussion}

In the present study, for the first time we assessed the genomewide lncRNA expression patterns in adriamycin resistant breast cancer cells using microarrays and explored their possible functions by analyzing their $c i s$-regulated mRNAs, as well as TF-regulated mRNAs.

We first identified dysregulated lncRNAs in adriamycinresistant MCF-7/ADM cells; these IncRNAs correlated with a list of dysregulated mRNAs. Because most of the lncRNAs in current databases have not yet been functionally annotated, we predicted their functions based on their correlated mRNAs. Chemoresistance is an important feature of cancer progression, so the lncRNAs dysregulated in chemoresistant MCF-7/ADM cells also showed functions associated with hallmarks of cancer progression (31). For instance, proteoglycans in cancer (KEGG 05205) are responsible for increased cancerous angiogenesis and provide a favorable microenvironment for cancer cells (32); and toll-like receptor signaling pathways (GO:0002224 and 0002756) provide cancer cells with sustained proliferative signals (33). Furthermore, the key feature of chemoresistant cancer cells is insensitivity to the cytotoxicity of chemotherapeutic agents. Such insensitivity could be achieved by a low efficacy of cellular drug transport, which may be associated with actin filament bundle assembly (GO:0051017) (34) and endocytosis (KEGG 04144) (35). Importantly, apoptosis inactivation and cell proliferation enhancement, whose pathways were frequently enriched in both the GO and KEGG pathways, not only support cancer growth and metastasis but also contribute to the insensitivity of cancer cells to chemotherapeutic agents. Therefore, being the 'mission critical' of cancer progression and chemoresistance regulated by various genetic and epigenetic mechanisms, we suggest apoptosis and cell proliferation may be still the main targets of regulation by 1 cRNAs.

Cis-action on target genes located at or near the same locus is one of the main mechanism by which lncRNAs regulate gene expression (36). We therefore identified genes whose expression was correlated with that of nearby lncRNAs. This analytical method greatly facilitated the identification of lncRNAs critical for chemoresistance. Based on the roles of lncRNAs in apoptosis and cell proliferation during chemoresistance, we then explored the possible mechanism of action of NONHSAT028712 in MCF-7/ADM and MCF-7/PTX cells because it may cis-regulate $\mathrm{CDK} 2$. The preliminary results strongly suggest an interaction between NONHSAT028712, CDK2, the cell cycle, and chemoresistance, and further studies are needed to clarify how NONHSAT028712 modulates expression of the nearby CDK2 gene; it may directly interact with the gene, facilitate the 3D folding of chromatin, or interact with other genetic (e.g. TFs) and epigenetic (e.g. microRNAs) regulators of the CDK2 gene (36).

LncRNAs frequently physically interact with TFs to regulate gene expression. We found that NONHSAT057282 and NONHSAG023333 were involved in chemoresistance. Then the IncRNA-TF interaction analysis suggests that these two lncRNAs may interact with ELF1 and E2F1 respectively, and subsequently modulated a group of chemoresistance-related genes such as GSTP1 (37,38), BTG3 (39), SOCS3 (40) and BRAC2 (41). Furthermore, among the identified lncRNA-TF interactions, ELF1 showed the highest enrichment frequency; 50 lncRNAs were significantly associated with this TF. ELF1 belong to the ETS transcription factorfamily, which is important for cancer progression (42) and breast cancer chemoresistance, as we demonstrated previously (25). ELF1 is associated with tumor angiogenesis (43), but its role in cancer chemoresistance has not been identified. Our results, thus, suggest ELF1 as a new participant in chemoresistance by potentially interacting with different lncRNAs. In future studies, mass spectrometry could be applied to confirm the lncRNA-TF interactions (44), and chromatin immunoprecipitation-based sequencing might be needed to verify the ELF1-related target genes (6). The TF ZEB1 also frequently interacted with lncRNAs. ZEB1 modulates the epithelial-mesenchymal transition pathway, which is essential for chemoresistance (45). Previous studies have shown that the epigenetic regulation of ZEB1 by microRNAs and DNA methylation effectively generates chemoresistance $(10,46)$, but few studies have explored the ZEB1-lncRNA interaction in chemoresistance. Therefore, considering the importance of ZEB1, it would be worthwhile to investigate the molecular mechanism by which lncRNAs mediate chemoresistance via ZEB1; here, we have provided several target lncRNAs that are likely to interact with ZEB1. Furthermore, 
other significantly enriched TFs, such as PBX3 (47) and E2F1 (48-50) are also involved in breast cancer progression; their interactions with lncRNAs may be important mechanisms by which they control gene expression and enhance chemoresistance.

In summary, we provide an overview of IncRNA regulation at the combined levels of lncRNA and gene expression in breast cancer chemoresistance, and systematically identify novel dysregulated targets in chemoresistant breast cancer cells. Experimental validation of specific interactions between lncRNAs and genes, and lncRNAs and TFs allowed us to identify the key players in chemoresistance, and decipher the underlining molecular mechanism of action of IncRNAs in cancer progression. Based on this analytical approach, we have shown the relationship and possible mechanism of action of several lncRNAs in the development of chemoresistance, suggesting that the analysis is precise and valuable for the support of future studies.

\section{Acknowledgements}

The present study was supported by the China National Natural Science Foundation (81572940 and 91439131 to X.M.; 31550006 to D.X.H.), the Natural Science Foundation for Distinguished Young Scholars of Jiangsu Province (BK20140004 to X.M.), the National High Technology Research and Development Program (863 Program) of China (SQ2015AA020948 to X.M.) and the Fundamental Research Funds for the Central Universities (JUSRP51311A and JUSRP51615B to X.M. and D.X.H.). We thank Professor I.C. Bruce for critical reading of the manuscript.

\section{References}

1. Marques AC, Hughes J, Graham B, Kowalczyk MS, Higgs DR and Ponting CP: Chromatin signatures at transcriptional start sites separate two equally populated yet distinct classes of intergenic long noncoding RNAs. Genome Biol 14: R131, 2013.

2. Louro R, Smirnova AS and Verjovski-Almeida S: Long intronic noncoding RNA transcription: Expression noise or expression choice? Genomics 93: 291-298, 2009.

3. Lam MT, Cho H, Lesch HP, Gosselin D, Heinz S, TanakaOishi Y, Benner C, Kaikkonen MU, Kim AS, Kosaka M, et al: Rev-Erbs repress macrophage gene expression by inhibiting enhancer-directed transcription. Nature 498: 511-515, 2013.

4. Li W, Notani D, Ma Q, Tanasa B, Nunez E, Chen AY, Merkurjev D, Zhang J, Ohgi K, Song X, et al: Functional roles of enhancer RNAs for oestrogen-dependent transcriptional activation. Nature 498: 516-520, 2013.

5. Vance KW and Ponting CP: Transcriptional regulatory functions of nuclear long noncoding RNAs. Trends Genet 30: 348-355, 2014.

6. Gupta RA, Shah N, Wang KC, Kim J, Horlings HM, Wong DJ, Tsai MC, Hung T, Argani P, Rinn JL, et al: Long non-coding RNA HOTAIR reprograms chromatin state to promote cancer metastasis. Nature 464: 1071-1076, 2010.

7. Ji P, Diederichs S, Wang W, Böing S, Metzger R, Schneider PM, Tidow N, Brandt B, Buerger H, Bulk E, et al: MALAT-1, a novel noncoding RNA, and thymosin beta4 predict metastasis and survival in early-stage non-small cell lung cancer. Oncogene 22: 8031-8041, 2003.

8. Qiu MT, Hu JW, Yin R and Xu L: Long noncoding RNA: An emerging paradigm of cancer research. Tumour Biol 34: 613-620, 2013.

9. He DX, Gu F, Gao F, Hao JJ, Gong D, Gu XT, Mao AQ, Jin J, Fu L and Ma X: Genome-wide profiles of methylation, microRNAs, and gene expression in chemoresistant breast cancer. Sci Rep 6: $24706,2016$.
10. Adam L, Zhong M, Choi W, Qi W, Nicoloso M, Arora A, Calin G, Wang H, Siefker-Radtke A, McConkey D, et al: miR-200 expression regulates epithelial-to-mesenchymal transition in bladder cancer cells and reverses resistance to epidermal growth factor receptor therapy. Clin Cancer Res 15: 5060-5072, 2009.

11. Chan YC, Khanna S, Roy S and Sen CK: miR-200b targets Ets-1 and is down-regulated by hypoxia to induce angiogenic response of endothelial cells. J Biol Chem 286: 2047-2056, 2011.

12. Feng X, Wang Z, Fillmore R and Xi Y: MiR-200, a new star miRNA in human cancer. Cancer Lett 344: 166-173, 2014.

13. Neves R, Scheel C, Weinhold S, Honisch E, Iwaniuk KM, Trompeter HI, Niederacher D, Wernet P, Santourlidis S and Uhrberg M: Role of DNA methylation in miR-200c/141 cluster silencing in invasive breast cancer cells. BMC Res Notes 3: 219, 2010.

14. Peter ME: Let-7 and miR-200 microRNAs: guardians against pluripotency and cancer progression. Cell Cycle 8: 843-852, 2009.

15. Lee JT: Epigenetic regulation by long noncoding RNAs. Science 338: 1435-1439, 2012.

16. Mercer TR and Mattick JS: Structure and function of long noncoding RNAs in epigenetic regulation. Nat Struct Mol Biol 20: 300-307, 2013.

17. Ma X, Cai Y, He D, Zou C, Zhang P, Lo CY, Xu Z, Chan FL, Yu S, Chen Y, et al: Transient receptor potential channel TRPC5 is essential for P-glycoprotein induction in drug-resistant cancer cells. Proc Natl Acad Sci USA 109: 16282-16287, 2012.

18. Li Y, Wang T, Li Y, Chen D, Yu Z, Jin L, Ni L, Yang S, Mao X, Gui Y, et al: Identification of long-non coding RNA UCA1 as an oncogene in renal cell carcinoma. Mol Med Rep 13: 3326-3334, 2016.

19. Wang XS, Zhang Z, Wang HC, Cai JL, Xu QW, Li MQ, Chen YC, Qian XP, Lu TJ, Yu LZ, et al: Rapid identification of UCA1 as a very sensitive and specific unique marker for human bladder carcinoma. Clin Cancer Res 12: 4851-4858, 2006.

20. Mourtada-Maarabouni M, Pickard MR, Hedge VL, Farzaneh F and Williams GT: GAS5, a non-protein-coding RNA, controls apoptosis and is downregulated in breast cancer. Oncogene 28: 195-208, 2009.

21. Panzitt K, Tschernatsch MM, Guelly C, Moustafa T, Stradner M, Strohmaier HM, Buck CR, Denk H, Schroeder R, Trauner M, et al: Characterization of HULC, a novel gene with striking up-regulation in hepatocellular carcinoma, as noncoding RNA. Gastroenterology 132: 330-342, 2007.

22. Braconi C, Kogure T, Valeri N, Huang N, Nuovo G, Costinean S, Negrini M, Miotto E, Croce CM and Patel T: microRNA-29 can regulate expression of the long non-coding RNA gene MEG3 in hepatocellular cancer. Oncogene 30: 4750-4756, 2011.

23. Khaitan D, Dinger ME, Mazar J, Crawford J, Smith MA, Mattick JS and Perera RJ: The melanoma-upregulated long noncoding RNA SPRY4-IT1 modulates apoptosis and invasion. Cancer Res 71: 3852-3862, 2011.

24. Ellis BC, Molloy PL and Graham LD: CRNDE: A long noncoding RNA involved in cancer, neurobiology, and development. Front Genet 3: 270, 2012

25. He D, Gu X, Jiang L, Jin J and Ma X: A methylation-based regulatory network for microRNA 320a in chemoresistant breast cancer. Mol Pharmacol 86: 536-547, 2014.

26. He DX, Gu XT, Li YR, Jiang L, Jin J and Ma X: Methylationregulated miRNA-149 modulates chemoresistance by targeting NDST1 in human breast cancer. FEBS J 281: 4718-4730, 2014.

27. Guttman M, Amit I, Garber M, French C, Lin MF, Feldser D, Huarte M, Zuk O, Carey BW, Cassady JP, et al: Chromatin signature reveals over a thousand highly conserved large noncoding RNAs in mammals. Nature 458: 223-227, 2009.

28. Marone M, Scambia G, Giannitelli C, Ferrandina G, Masciullo V, Bellacosa A, Benedetti-Panici P and Mancuso S: Analysis of cyclin $\mathrm{E}$ and $\mathrm{CDK} 2$ in ovarian cancer: Gene amplification and RNA overexpression. Int J Cancer 75: 34-39, 1998.

29. Opyrchal M, Salisbury JL, Iankov I, Goetz MP, McCubrey J, Gambino MW, Malatino L, Puccia G, Ingle JN, Galanis E, et al: Inhibition of Cdk2 kinase activity selectively targets the CD $44^{+} /$ $\mathrm{CD} 24^{+} /$Low stem-like subpopulation and restores chemosensitivity of SUM149PT triple-negative breast cancer cells. Int J Oncol 45: 1193-1199, 2014.

30. Gerstein MB, Kundaje A, Hariharan M, Landt SG, Yan KK, Cheng C, Mu XJ, Khurana E, Rozowsky J, Alexander R, et al: Architecture of the human regulatory network derived from ENCODE data. Nature 489: 91-100, 2012. 
31. Hanahan D and Weinberg RA: Hallmarks of cancer: the next generation. Cell 144: 646-674, 2011.

32. Iozzo RV and Sanderson RD: Proteoglycans in cancer biology, tumour microenvironment and angiogenesis. J Cell Mol Med 15: 1013-1031, 2011.

33. Rakoff-Nahoum S and Medzhitov R: Toll-like receptors and cancer. Nat Rev Cancer 9: 57-63, 2009.

34. Fu D and Roufogalis BD: Actin disruption inhibits endosomal traffic of P-glycoprotein-EGFP and resistance to daunorubicin accumulation. Am J Physiol Cell Physiol 292: C1543-C1552, 2007.

35. Gottesman MM: Mechanisms of cancer drug resistance. Annu Rev Med 53: 615-627, 2002.

36. Guil S and Esteller M: Cis-acting noncoding RNAs: Friends and foes. Nat Struct Mol Biol 19: 1068-1075, 2012

37. Townsend DM and Tew KD: The role of glutathione-S-transferase in anti-cancer drug resistance. Oncogene 22: 7369-7375, 2003.

38. Traverso N, Ricciarelli R, Nitti M, Marengo B, Furfaro AL, Pronzato MA, Marinari UM and Domenicotti C: Role of glutathione in cancer progression and chemoresistance. Oxid Med Cell Longev 2013: 972913, 2013.

39. Yu J, Zhang Y, Qi Z, Kurtycz D, Vacano G and Patterson D: Methylation-mediated downregulation of the B-cell translocation gene 3 (BTG3) in breast cancer cells. Gene Expr 14: 173-182, 2008.

40. Ru P, Steele R, Hsueh EC and Ray RB: Anti-miR-203 upregulates SOCS3 expression in breast cancer cells and enhances cisplatin chemosensitivity. Genes Cancer 2: 720-727, 2011.

41. Wang W and Figg WD: Secondary BRCA1 and BRCA2 alterations and acquired chemoresistance. Cancer Biol Ther 7 : 1004-1005, 2008.
42. Seth A and Watson DK: ETS transcription factors and their emerging roles in human cancer. Eur J Cancer 41: 2462-2478, 2005.

43. Huang X, Brown C, Ni W, Maynard E, Rigby AC and Oettgen P: Critical role for the Ets transcription factor ELF-1 in the development of tumor angiogenesis. Blood 107: 3153-3160, 2006.

44. Li Z, Chao TC, Chang KY, Lin N, Patil VS, Shimizu C, Head SR, Burns JC and Rana TM: The long noncoding RNA THRIL regulates TNF $\alpha$ expression through its interaction with hnRNPL. Proc Natl Acad Sci USA 111: 1002-1007, 2014.

45. Lee JM, Dedhar S, Kalluri R and Thompson EW: The epithelialmesenchymal transition: new insights in signaling, development, and disease. J Cell Biol 172: 973-981, 2006.

46. Pieraccioli M, Imbastari F, Antonov A, Melino G and Raschellà G: Activation of miR200 by c-Myb depends on ZEB1 expression and miR200 promoter methylation. Cell Cycle 12: 2309-2320, 2013

47. Han HB, Gu J, Zuo HJ, Chen ZG, Zhao W, Li M, Ji DB, Lu YY and Zhang ZQ: Let-7c functions as a metastasis suppressor by targeting MMP11 and PBX3 in colorectal cancer. J Pathol 226: 544-555, 2012.

48. Knoll S, Emmrich S and Pützer BM: The E2F1-miRNA cancer progression network. Adv Exp Med Biol 774: 135-147, 2013.

49. Frietze S, Lupien M, Silver PA and Brown M: CARM1 regulates estrogen-stimulated breast cancer growth through up-regulation of E2F1. Cancer Res 68: 301-306, 2008.

50. Louie MC, Zou JX, Rabinovich A and Chen HW: ACTR/AIB1 functions as an E2F1 coactivator to promote breast cancer cell proliferation and antiestrogen resistance. Mol Cell Biol 24: 5157-5171, 2004. 